\title{
GAS AMPLIFICATION IN PROPORTIONAL COUNTERS
}

\author{
By S. RAMAKRISHNA \\ (Department of Physics, Indian Institute of Science, Bangalore-12) \\ Received September 19, 1959 \\ (Communicated by Prof. R. S. Krishnan, F.A.sc.)
}

\section{INTRODUCTION}

SEVERAL workers have studied the gas amplification factor and its variation with field strength for proportional counters. Rossi and Staub (1948) have given data for various gas fillings at different pressures. Rose and Korff (1941) have theoretically derived the following expression for the gas amplification factor in terms of measurable quantities like the wire diameter, the pressure of the gas inside the counter and the specific ionisation coefficient,

$$
\mathrm{A}=\exp \cdot(a \cdot \mathrm{N} \cdot \mathrm{C} \cdot \mathrm{V} \cdot r)^{ \pm}\left\{\left(\frac{\mathrm{V}}{\mathrm{V}_{t}}\right)^{ \pm}-1\right\} \text {. }
$$

Measurements of gas amplification factors have been made by Rose and Korff (1941) and Rossi and Staub (1948) for testing the validity and scope of the above equation and also to determine which of the assumptions that have been made in the derivation of the theory are not completely justifiable. This paper describes measurements of gas amplification factor as a function of voltage under different conditions of pressure, gas filling and concentrations of the quenching agent.

\section{Experimental Details}

The counter employed for the measurements was made of a copper cathode $10 \mathrm{~cm}$. long and $3.5 \mathrm{~cm}$. in diameter, with a 3 mil. central wire. Mono-energetic alpha rays from a $\mathbf{P O}^{210}$ source were used for the experiments.

The measurement of gas amplification factor was carried out according to the method used by Rossi and Staub (1948). Essentially, their method consisted in the use of an oscilloscope for the measurement of the pulse heights at various counter voltages. The counter was then operated in the ionisation chamber region and the electronic amplification necessary to produce an output pulse of the same height as before was taken as a measure of the gas amplification factor at the particular voltage. A linear 
amplifier based on a circuit given by Elmore and Sands (1949) was constructed and used for the present measurements. The amplifier linearity for input pulses ranging from $100 \mu_{v}$ (which was the minimum detectable signal above the noise level) to about $1 \mathrm{~V}$ (corresponding to a ratio of $1: 10,000$ in input pulse heights) was checked by standard methods.

The counter was filled with the gas to be studied and placed in a wellshielded box to avoid pick-up. The pulses from the counter were displayed on the oscilloscope screen after amplification. When the counter was operating in the ionisation chamber region, which is indicated by the pulse height remaining appreciably constant with changes in applied voltage, the pulse height on the oscilloscope screen was measured. The voltage was then increased in steps and the pulse height was adjusted to be the same as when the counter was operating in the ionisation chamber region, by means of the attenuator. The amplification factor corresponding to a given voltage was obtained by noting the attenuation that was introduced.

Ordinarily, such measurements would be limited by the setting in of space-charge effects. It has been shown (Venkatasubramanian, 1957) that space-charge effects become considerable at values of $\mathrm{A}$ as low as $10^{3}$ for alpha particles whose entire track is assumed to be confined within the counting volume. Rossi and Staub have also confined their values to less than $10^{3}$.

In the following experiments, this upper limit has been pushed up by making the alpha particle spend only part of its track inside the counting volume. The total ionisation is consequently less and hence space-charge limitations set in at higher values of A. However, even this method cannot be carried too far, since if too small a portion of the track is spent inside the counter the error in the accurate computation of the pulse size increases. The author has therefore given values of $A$ up to about 2,000 only.

\section{RESULTS}

Experiments were carried out on $10 \%$ and $25 \%$ argon-ethane and argon$\mathrm{CO}_{2}$ mixtures. The results obtained are graphically represented in Figs. 1-3. The critical voltages given in Table $I$ indicate the onset of the Geiger region. It is seen that the gas amplification values range up to 1,000 for the argon-ethane mixture and the argon- $\mathrm{CO}_{2}$ mixture. The gas amplification curves show some interesting features and these are discussed in Table $I$.

\section{DisCusSION}

(a) Effect of Pressure.-Figure 1 gives the variation of A with the applied voltage for two different gas pressures for argon-ethane counters. The 


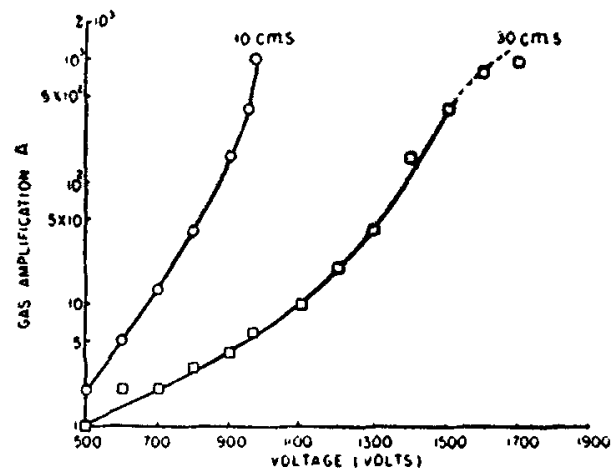

Fio. 1

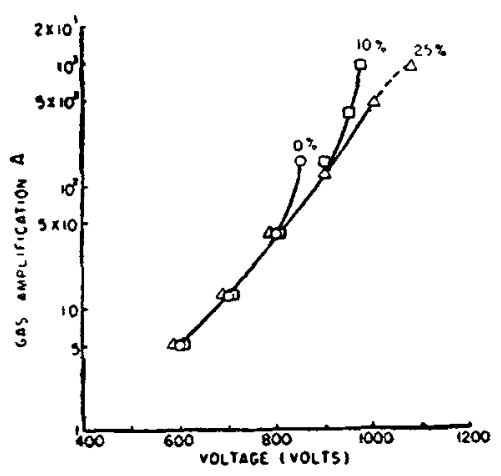

FIG. 2

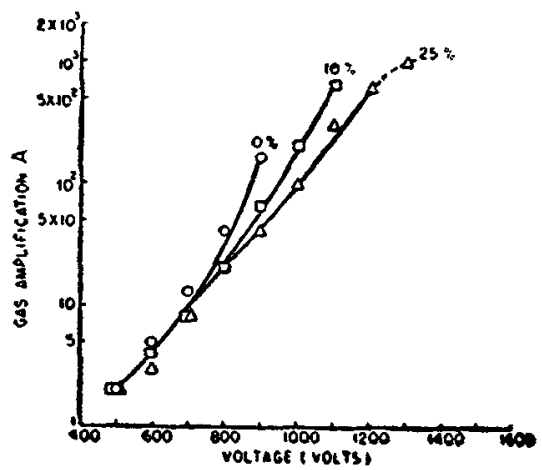

Fig. 3

Fros. 1-3. Fig. 1. Gas Amplification vs. Voltage for Argon-Ethane Filling. Fig. 2. Ga 8 Amplification vs. Voltage for Argon-Ethane Filling. Fig. 3. Gas Amplification ys. Voltage for Argon- $\mathrm{CO}_{2}$ Filling.

\section{TABLE I}

Gas filling

Pressure

Argon + quenching agent $10 \mathrm{~cm}$.

\begin{tabular}{|c|c|c|c|}
\hline \multirow[t]{2}{*}{ No. } & \multirow{2}{*}{$\underset{\%}{\text { Quenching Agent }}$} & \multicolumn{2}{|c|}{$\begin{array}{c}\text { Critical voltage } \\
\text { volts }\end{array}$} \\
\hline & & $\mathrm{CO}_{2}$ & $\mathrm{C}_{2} \mathrm{H}_{6}$ \\
\hline 1 & 0 & 880 & 880 \\
\hline 2 & 10 & 1,200 & 980 \\
\hline 3 & 25 & 1,420 & 1,100 \\
\hline
\end{tabular}


curves drawn by the author are in general agreement with the results of other workers. The increase in value of $A$ at lower pressures is a result of a decrease in the threshold voltage. At higher pressures, the increase in A with voltage is less rapid since $a / p$ does not increase as fast as it does at lower pressures.

(b) Effect of Quenching Agent.-It is well known (Trost, 1937) that the addition of a little quenching agent like ethyl alcohol results in a reduction of photo-emission and secondary electron emission in a Geiger counter. The action of the quenching agent in a proportional counter is also similar. The only difference is that in the former it prevents the setting in of a continuous discharge, while in the latter it prevents the spread of the discharge along the central wire.

The manner in which a quenching agent such as ethyl alcohol or methane prevents photo-emission and secondary electron emission has been described in detail by Korff and Present (1944). The de-excitation of excited atoms or molecules in the counter, by means of photon emission, causes a spread in the discharge. This is prevented by the organic quenching gas since these normally have a continuous absorption band in the ultra-violet, in which region the photon energies normally fall. Diatomic and monatomic molecules, however, have stable and discrete electronic states and de-excite predominantly by photo-emission or fluorescence. Secondary electron emission takes place if the energetic ions are able to approach the cathode to within a critical distance (Massey, 1930) usually of the order of $10^{-7} \mathrm{~cm}$. This is prevented by the quenching agent by a charge exchange process,

$$
\mathrm{A}^{+}+\left(\mathrm{C}_{2} \mathrm{H}_{5}\right)-\mathrm{A}+\left(\mathrm{C}_{2} \mathrm{H}_{5}\right)^{+}
$$

resulting in polyatomic molecules and not argon atoms to be incident on the cathode. These pre-dissociate rather than cause secondary emission, and even if in some cases they do liberate electrons, they are degraded in energy and are not capable of causing large avalanches.

In view of the above considerations, it is evident that both ethane and carbon dioxide will be suitable as quenching agents. This is borne out by the fact that with only argon as the filling gas, the experimental curve departs from the theoretical predictions (by showing a faster rise than is expected) at gas amplification factors as low as 100 , while with the addition of ethane or carbon dioxide, the exponential rise is maintained up to values of 2,000 for A. Argon-ethane counters have the desirable property of lower operating voltages, but the gas amplification characteristic of an argon$\mathrm{CO}_{2}$ counter is more satisfactory in the sense that the rate of increase of $\mathrm{A}$ with the applied voltage is not very rapid. Thus, both ethane and carbon 
dioxide can be used with advantage as quenching vapours in proportional counters.

(c) Effect of Concentration.-At low gas amplification factors and at low concentrations of the quenching agent, there is hardly any effect at all on the gas amplification characteristic. Since none of the processes for the prevention of which the quenching agent is added are in operation at low values of $A$, this is to be expected. At higher values of $A$, however, the quenching agent prevents the pass-over into the Geiger region, for reasons given above. Table I gives the voltages at which Geiger discharge sets in, for various concentrations of the quenching agent. Carbon dioxide is found to be more effective in this process since its ionisation potential is nearer to that of argon and hence electron transfer is expected to be more efficient. A further reason is that in accordance with the theory, the addition of appreciable quantities of quenching agent changes the value of the term $a$, which is a characteristic of each gas (Curran and Craggs, 1949) in the expression for the gas amplification factor.

Quantitatively, as seen from the curves, the slope at low gas amplification factors does not alter appreciably for concentrations up to about $30 \%$ in the case of ethane and $20 \%$ for $\mathrm{CO}_{2}$. The characteristics actually coincide in the low gas amplification region, within the experimental accuracy. This has also been observed by Colli and Facchini (1952) for low concentrations of $\mathrm{CO}_{2}$.

\section{SUMMARY}

The variation of gas amplification with applied voltage is an important characteristic of a proportional counter. Results of studies on gas amplification for gas fillings of argon quenched with ethane and carbon dioxide are given. The effects of $(a)$ pressure, $(b)$ quenching agent and (c) concentration on $\mathrm{A}$ are discussed briefly.

\section{ACKNOWLEDGEMENT}

I thank Professor R. S. Krishnan for guidance and suggestions. I also thank Dr. V. S. Venkatasubramanian for helpful discussions.

\section{REFERENCES}

1. Colli, L. and Facchini, U. .. Physica, 1952, 18, 1272.

2. Curran, S. C. and Craggs, Counting Tubes, Butterworths, 1949. J. D.

3. Elmore and Sands $\quad$.. Electronics-Experimental Techniques, McGraw-Hill, 1949. 
4. Korff, S. A. and Present, Phys. Rev., 1944, 65, 274.

R. D.

5. Massey, H. S. W. _. Proc. Camb. Phil. Soc., 1930, 26, 386.

6. Rose, M. E. and Korf, S. A. Phys. Rev., 1941, 59, 850.

7. Rossi, B. and Staub, H. .. Ionisation Chambers and Counters, McGraw-Hill, 1948.

8. Trost, A. $\quad$.. Zeits. f. Phys., 1937, 105, 399.

9. Venkatasubramanian, V. S. Jour. Ind. Inst. Sci., 1957, 39, 125. 